\title{
Seasonal forecasting of tropical cyclone activity in the coastal region of Vietnam using RegCM4.2
}

\author{
Tan Phan-Van*, Long Trinh-Tuan, Hai Bui-Hoang, Chanh Kieu
}

Department of Meteorology, VNU Hanoi University of Science, 334 Nguyen Trai, Thanh Xuan, Hanoi, Vietnam

\begin{abstract}
This study presents an experimental seasonal forecast of tropical cyclone (TC) activity for Vietnam's coastal region during the 2012-2013 typhoon seasons, using the Regional Climate Model (RegCM, version 4.2) to downscale the global Climate Forecasting System (CFS) forecast. Using an improved vortex tracking algorithm that detects vortex centers efficiently, RegCM reasonably forecasts the general distribution of TC counts in time as well as the TC track pattern during the entire experimental period from February to July 2012 and 2013, despite significant underestimation of the TC counts in the global CFS forecasts that are used as initial and lateral boundary conditions for the RegCM model. Further examination of the storm activity in the Vietnam East Sea that directly influences Vietnam's coastal region shows, however, that RegCM tends to overestimate the TC frequency in this sub-region compared to observation. This suggests that direct applications of the RegCM model for seasonal forecasts of TC activity in Vietnam's coastal region has a significant bias that will need to be corrected before the model can provide useful information.
\end{abstract}

KEY WORDS: Seasonal forecasting - Tropical cyclone $\cdot$ TC detection $\cdot$ Vietnam $\cdot$ Dynamical downscaling

Resale or republication not permitted without written consent of the publisher

\section{INTRODUCTION}

Seasonal forecasts of tropical cyclone (TC) activity play a critical role for risk management and economic assessments. This is particularly the case for regions with high population densities along coastlines that are under threat of landfalling TCs. With a coastline of $>3000 \mathrm{~km}$, Vietnam is vulnerable to the impact of TCs in the Northwestern Pacific (WPAC) basin. Of about 28 typhoons originating in the WPAC basin each year, about 10 move over the Vietnam East Sea (VES; also called the South China Sea). Of these, 4 to 6 storms have a direct influence on Vietnam's coastline, causing a significant impact on society and economic activity. Seasonal real-time forecasts of TCs in the WPAC basin, especially those confined within the VES to the east of Vietnam, are still challenging because of the difficulties in quantifying TC behavior in such a limited region (Chan et al. 1998, Chan, 2008).
Roughly speaking, there are 3 main approaches to seasonal TC forecasting: (1) a dynamical approach in which numerical climate models are used to predict TC formation and development, (2) a statistical approach with some assumed empirical relationship between TC activity and a set of selected predictors; and (3) a combination of the statistical and dynamical approach, the so-called statistical-dynamical method (see e.g. Chan et al. 2001, Camargo \& Barnston 2009, Klotzbach \& Gray 2009, Vecchi et al. 2011, Kim et al. 2012, Lu et al. 2013). Of the 3, statistical methods appear to be dominant in most seasonal forecasts, due to their relatively higher skill and inexpensive computation compared to the coarse resolution climate models (Klotzbach 2007, Vitart et al. 2010, Yeung \& Chan 2012).

Recent advances in climate modeling have resulted in a new generation of climate models that could provide skillful seasonal forecasts of TC activity, comparable in skill to the statistical forecasts, especially 
when used as input for statistical-dynamical models. In particular, regional climate models play an important role in climate projections under different climate change scenarios, which statistical models could not achieve alone (Vitart et al. 2010, Vecchi et al. 2011, Kim et al. 2012). Nonetheless, inherent uncertainties in the model dynamics and representations of physical and thermodynamic feedbacks, as well as inaccurate boundaries, render climate models in general less accurate than the statistical approach at long forecast lead times. In fact, the most reliable seasonal forecasts of TC activity still rely on a statistical approach rather than on the pure dynamical climate models (Klotzbach \& Gray 2009, Vitart et al. 2010).

Given current model uncertainties, seasonal TC forecasting based on global or regional climate models has been so far mostly experimental. Operational seasonal forecasts provided by the National Centers for Environmental Prediction (NCEP) Climate Forecast System (CFS) are perhaps the most used seasonal products available in real time (Saha et al. 2010). The CFS system consists of 6 mo forecasts available daily with 6-hourly outputs, and serves a wide range of downstream seasonal applications ${ }^{1}$. A growing number of studies of the CFS forecasting system demonstrate its capability in seasonal forecasting, including forecasts of ENSO variability and precipitation over the tropical region (Kirtman \& Min 2009, Wang et al. 2010, Sooraj et al. 2012), intraseasonal oscillation and winter persistent inversions (Gillies et al. 2010), SST anomalies (Wu et al. 2009), and extreme climate events (Becker et al. 2013).

Because of their coarse resolution and simplified physics, direct applications of the CFS products to regional climate forecasts are difficult in practice. Thus, dynamical downscaling of the CFS products with a regional climate model is necessary to enhance the regional characteristics. This downscaling is especially vital for TC forecasting because TC intensity and development depends strongly on the model resolution and model physics (Bengtsson et al. 2007, Vitart et al. 2010, Yeung \& Chan 2012, Strachan et al. 2013, Vecchi et al. 2014). As a result, using only the CFS forecasts would provide an unreliable count of TC numbers. A recent study by Yeung \& Chan (2012) demonstrated the necessity of regional downscaling in TC seasonal forecast for the WPAC basin,

\footnotetext{
1'Inventory and support for this CFS dataset can be found on the NCEP climate forecast system (CFS) products website at: http://nomads.ncep.noaa.gov/pub/data/nccf/com/cfs/ $\mathrm{prod} / \mathrm{cfs}$
}

using the Regional Climate Model (RegCM) to dynamically downscale the ERA40 reanalysis. Their study showed that RegCM is capable of reproducing the climatology of the TC activity in the WPAC basin fairly well in terms of spatial and temporal distribution during the 1982-2001 period. Nevertheless, Yeung and Chan's study focused more on the general hindcasting of TC genesis and development over the WPAC basin with the ERA-40 dataset, rather than real-time seasonal forecasting. Therefore it has only limited application to seasonal forecasting of TCs along Vietnam's coastal region, defined as a zone extending to the meridian of $120^{\circ} \mathrm{E}$ from Vietnam's coastal baseline (hereinafter referred to as the VNC area).

With the CFS products available in real-time, it is of interest to examine how they can be applied to regional TC forecasting. In this study, we examine the capability of the RegCM model in downscaling the CFS products for seasonal forecasts of TCs in the VES that could potentially influence Vietnam's coastline. Although there have been some studies of seasonal typhoon forecasting for the WPAC basin (Chan et al. 1998, 2001, Lu et al. 2010, Kim et al. 2012, Yeung \& Chan 2012), explicit forecasts of TC activity for the VNC area are still inadequate. In this study, we present a modified vortex tracking algorithm that is designed specifically for detecting TC-like vortices from the RegCM model output. This vortex track algorithm is needed to improve the capability of the RegCM model in forecasting of TC activity, due to RegCM's coarse resolution.

\section{EXPERIMENTAL DESCRIPTION}

\subsection{Model}

In this study, version 4 of the Regional Climate Model (RegCM4.2) was used to provide experimental real-time seasonal forecasts of TC activity for the 2012-2013 seasons in the WPAC basin, with the main focus on TCs that are most influential to the VNC area. The RegCM model was based on the FourthGeneration Mesoscale Model developed in the 1980s (Dickinson et al. 1989, Giorgi \& Bates 1989, Giorgi et al. 1993a,b). RegCM4.2 was a hydrostatic version in the vertical sigma coordinate that shared many features of the hydrostatic version of the fifth-generation Pennsylvania State University-National Center for Atmospheric Research Mesoscale Model (MM5; Grell et al. 1994). Several fundamental differences compared to MM5 include the land surface scheme, the radiation 
parameterizations, and convective schemes (Elguindi et al. 2004). Recent upgrades of the RegCM model included a number of new physics packages that were based on physics schemes of the Community Climate Model, including new aerosol radiative transfer calculations, a new prognostic equation for cloud water, and a new parameterization of surface land use (see, e.g. Pal et al. 2007, Solmon et al. 2008, Elguindi et al. 2011, Giorgi \& Anyah 2012, Giorgi et al. 2012 for more information).

In all experimental real-time forecasts, RegCM4.2 is configured with a horizontal grid spacing of $36 \mathrm{~km}$, 18 vertical sigma levels, and the model top at $10 \mathrm{hPa}$. The model domain is centered at $20^{\circ} \mathrm{N}, 140^{\circ} \mathrm{E}$, and consists of 146 grid points in the east-west direction and 288 grid points in the north-south direction, spanning an area from 100 to $180^{\circ} \mathrm{E}$ and $5^{\circ} \mathrm{S}$ to $40^{\circ} \mathrm{N}$ (Fig. 1). This domain is sufficiently large to capture not only storms formed in the VES, but also most of TCs formed in the far-east region of the Philippines archipelago that could travel to the region. The model time step was set to 60 s. Model physics schemes used in this study consist of (1) the Community Climate Model Version 3 (CCM3) radiative transfer scheme, (2) the Biosphere Atmosphere Transfer Scheme (BATS) land surface scheme, and (3) the Grell-Arakawa-Schubert cumulus parameterization scheme (Grell-AS). A sensitivity study of the RegCM model (Phan et al. 2009) suggested that these above schemes are adequate for simulating climate in Vietnam and Southeast Asia. Therefore, these parameterization schemes were chosen for the seasonal $\mathrm{TC}$ forecast in all of our experiments.

\subsection{Real-time experiment}

The experiments were conducted during the 2012-2013 typhoon seasons using the RegCM4.2 model to downscale the global CFS products, which were provided in real-time by NCEP at the horizontal resolution of $1 \times 1^{\circ}$ (RegCM_CFS1.0). The experiments were designed with the main focus on the 6 mo forecasts of TC activity, and were configured with a single domain as mentioned above. Forecasts began at 00:00 h UTC January 1 2012 and were updated every $7 \mathrm{~d}$ thereafter (i.e. there were four 6 mo forecasts conducted in each month). Lateral boundary conditions including the SST were updated every $6 \mathrm{~h}$ from the CFS forecasts. Each 6 mo forecast generated 6-hourly 3dimensional output consisting of horizontal wind, potential temperature, geopotential height on the pressure surfaces, and the sea level pressure. The output was subsequently post-processed by a modified vortex tracking program that detected and followed any vortex within the model domain. The real-time experiments were carried out during a 4 mo period from February to May in both 2012 and 2013 to generate 6 mo forecasts of TC activity in the WPAC basin and the VNC region (i.e. from March to August, April to September, May to October, and from June to November in 2012 and 2013). These forecasts supported risk management and natural disaster prevention actions by the Vietnam National Hydro-meteorological Service, for which we were responsible.

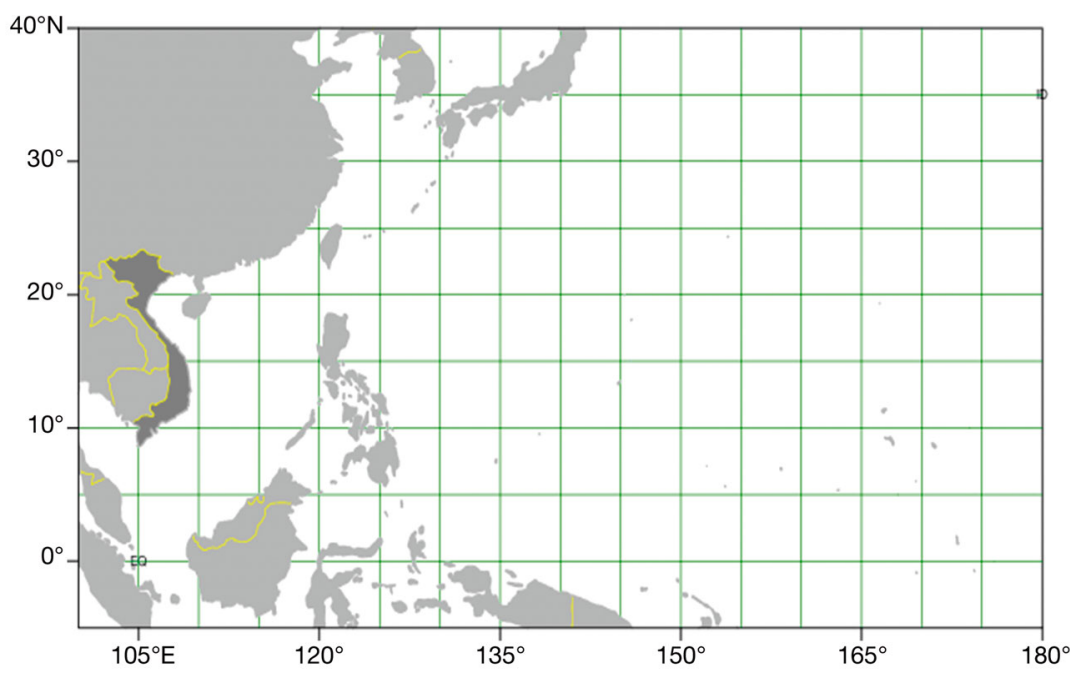

Fig. 1. Model domain configuration of the RegCM4.2 for experimental real-time forecasts of tropical cyclone (TC) activity for Vietnam's coastal region in the 2012-2013 seasons

\subsection{Dataset}

The primary data used in this study consists of (1) NCEP Climate Forecast System Reanalysis (CFSR) data for the period 1995-2010, and (2) NCEP CFS Version 2 real-time forecast data for the period 20122013. These datasets are available in GRIB2 format with a 6-hourly interval. Both the CFS and the CFSR gridded data are provided 4 times per day at the synoptic times of 00:00, 06:00, 12:00, and 18:00 h UTC. Note that the CFSR datasets are provided at 2 resolutions, i.e. $0.5 \times 0.5^{\circ}$ (CFSR0.5) and $2.5 \times 2.5^{\circ}$ (CFSR2.5), whereas the CFS real-time forecast datasets are archived on a $1 \times 1^{\circ}$ grid (CFS1.0), which could allow for the representation of the TC circulation and steering flow to some 
degree. Of course, the TC intensity and inner core structure are barely represented at these coarse resolutions, and therefore dynamical downscaling of the CFS products is needed to better capture the TC development and multi-scale interaction (Walsh \& Ryan, 2000, Strachan et al. 2013).

An additional dataset used to verify the TC frequency in the WPAC basin for both the baseline and real-time experiments is the TC best track data archived by Unisys Weather Information Systems (Unisys ${ }^{2}$ ) during the 1995-2013 period. This dataset contains the latitudes and longitudes of storm centers, storm lifetime, and storm intensity, and it is divided into different basins. This Unisys dataset is used for all the verifications in this study. Although there are several different databases from different agencies such as those maintained by Joint Typhoon Warning Center (JTWC) or the Japan Meteorological Agency, they are not consistent in terms of the exact storm locations or intensity (Knapp \& Kruk 2010). Nevertheless, these datasets are reliable in terms of the number of TCs, the intensity phases as well as the general track patterns. Since this study focuses mostly on the TC frequency and seasonal variations, such discrepancies in TC absolute intensity should have minimum impact on our analysis. Thus, the Unisys dataset can be expected to provide an adequate basis for use in this study.

\section{IMPROVED VORTEX TRACKING ALGORITHM}

With a typical horizontal resolution around $1 \times 1^{\circ}$ in most global climate models, a model vortex tends to exhibit few signals of the central temperature anomaly (warm core), the minimum sea level pressure, or the maximum surface wind speed (Bengtsson et al. 1995, Walsh 1997, Walsh \& Watterson 1997, Yeung \& Chan 2012, Strachan et al. 2013). For regional climate models with higher resolution, storm circulations are better represented, but model representations are still not comparable to the actual storms in terms of TC size and intensity. Therefore, an efficient vortex tracking algorithm is essential in order to reliably detect TC vortices from the model products.

In general, a vortex tracking algorithm examines a variety of fields including vorticity, surface wind, temperature at particular pressure levels, and the minimum sea level pressure (Bengtsson et al. 1995, Walsh 1997, Walsh \& Watterson 1997, Nguyen \&

${ }^{2}$ The Unisys dataset is available at http://weather.unisys.com
Walsh 2001, Yeung \& Chan 2012). Nevertheless, Camargo \& Zebiak (2002) showed that these parameters may sometimes capture local disturbances instead of proper TCs. They concluded that a tracking algorithm may need to be modified for different regions, model dynamics, or model resolution. Therefore, the threshold values are not universal and need to be tuned in properly for each specific model application.

In this study, we modified a version of the vortex tracking algorithm proposed by Walsh (1997) for our purpose of tracking vortices in the VNC region. In contrast to the original method that emphasizes on the vorticity parameter, our method considers a wider range of criteria. Our modified tracking algorithm fulfils 2 requirements: (1) the tracking method has to detect storms with at least tropical depression strength as well as all typical TC characteristics, not only in the open ocean but also close to coastlines; and (2) it must have the capability to distinguish one vortex from the other nearby so that the total TC count is computed correctly. While there are several different methods for tracking TC-like vortices in weather forecasting models, the main difficulty when using regional climate models is that their relatively low resolutions are not adequate to capture the TC characteristics of interest (see e.g. Walsh \& Ryan 2000). This limitation is compounded by the simplified model physics that is used in climate models to integrate data efficiently over a long period of time.

Our modified vortex tracking algorithm consists of 2 main phases: a detection phase and a tracking phase. In the detection phase, the model outputs are interpolated from the model (sigma) levels to 4 standard isobaric levels at 850, 700, 500 and $300 \mathrm{hPa}$. For the tracking phase, the following steps are carried out at each instant of model output:

(1) At each time step, a grid point is checked to see if its relative vorticity is a local maximum and has a value that is greater than a given threshold. The local maximum is identified by checking if the vorticity is larger than the vorticity of the 4 adjacent points in meridional and zonal directions. If the grid point satisfies this condition, a candidate for storm vortex center is marked.

(2) If a candidate grid point is found, the minimum sea level pressure within a radius of $250 \mathrm{~km}$ from the candidate grid point is searched using the downhill method combined with 2-dimensional spline interpolation. The location of the minimum sea level pressure after this step does not necessarily coincide with any model grid point because of the interpolation.

(3) If a minimum sea level pressure is found, other indicators are used to determine if this is a storm 
center. The following criteria are employed: (i) The minimum sea level pressure anomaly (DP), defined as the difference between the storm center pressure $\left(\mathrm{P}_{\text {center }}\right)$ and the environment pressure $\left(\mathrm{P}_{\text {env }}\right)$, is smaller than a given threshold; (ii) the core temperature anomaly (DT), calculated as a weighted average of temperature anomaly at isobaric levels, must be positive; and (iii) the outer core wind strength (OCS) has to be greater than a given value, which is best tuned for each specific model configuration and resolution.

(4) If all of the above criteria are satisfied, the location of the minimum sea level pressure obtained as described above is considered to be the center of a TC vortex. Since the detection is performed at each archive interval (every $6 \mathrm{~h}$ ), it is important that the detection process be able to distinguish whether the newly found center belongs to an old vortex from the previous archive interval or is the center of a new storm. This is done by checking the existence of any storm at the current time and the previous time within a domain of radius $250 \mathrm{~km}$ around the current center vortex. Assuming that the distance between 2 TCs is no less than $250 \mathrm{~km}$, this procedure should eliminate virtually all binary vortex situations. The processes are then repeated for the next cycle until the end of the searching period.

Note that OCS is defined as an average of the tangential wind speed at 36 points on 4 circles within an annulus domain between 2 circles of radii 1 and $2.5^{\circ}$ (Weatherford \& Gray 1988) (Fig. 2a). The average on these concentric points is calculated by interpolating the wind field from the model native grid to the cylindrical coordinate using the spline method. Similarly, calculations of other field anomalies such as DP or DT are done by subtracting the value of the field at the vortex center from the average of 8 points on a circle with radius of $2.5^{\circ}$ from the center (Fig. 2b). The weights for calculating DT are $0.4,0.3,0.2$, and 0.1 at isobaric levels of $300,500,700$ and $850 \mathrm{hPa}$ respectively. In contrast to the original algorithm by Walsh (1997), our modified algorithm puts more weight on OCS as the primary criterion to distinguish between a true TC circulation and spurious centers associated with high vorticity anomalies or a spurious low pressure area related to steep topography. Sensitivity experiments with different thresholds of vorticity revealed that the original algorithm by Walsh tends to produce too many TC centers along the Philippines archipelago, over land, or near coastlines where vorticity has some artificial local point-like maximum. Thus, the vorticity criterion is relaxed in our algorithm to eliminate those multiple unrealistic vortex centers near the coastal zone.

As a demonstration of the new tracking algorithm, Fig. 3 shows the mean bias error and the root mean square errors of the TC counts detected within the 1995-2010 period for 9 different sets of vortex tracking thresholds. Nine different combinations of vortex tracking criteria including the relative vorticity, OCS, and DP are used to select optimum criteria that provide best-fit TC counts compared to observation. A
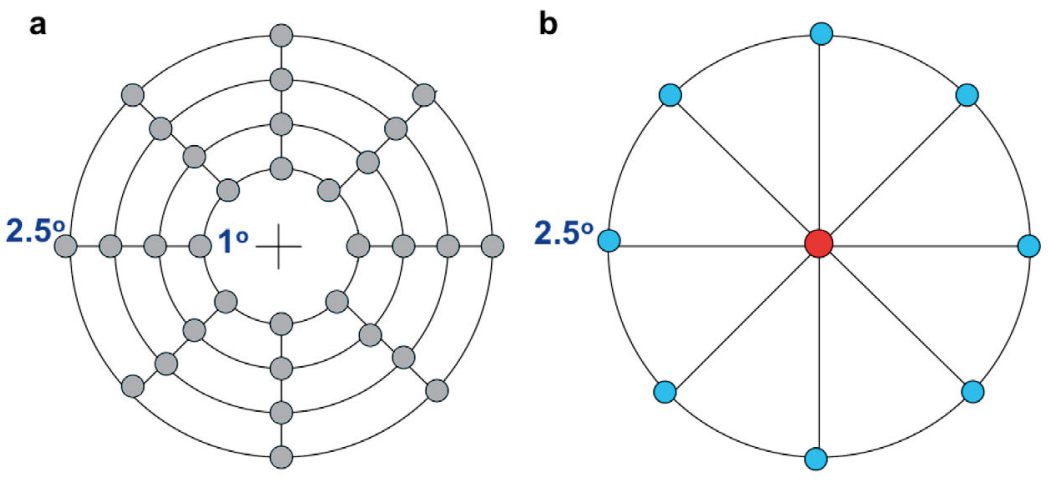

Fig. 2. Grid points (circles) in the cylindrical coordinate for calculating (a) the outer core wind and (b) the tropical cyclone (TC) warm core anomaly in the new vortex tracking algorithm. Cross and red point indicate the TC center
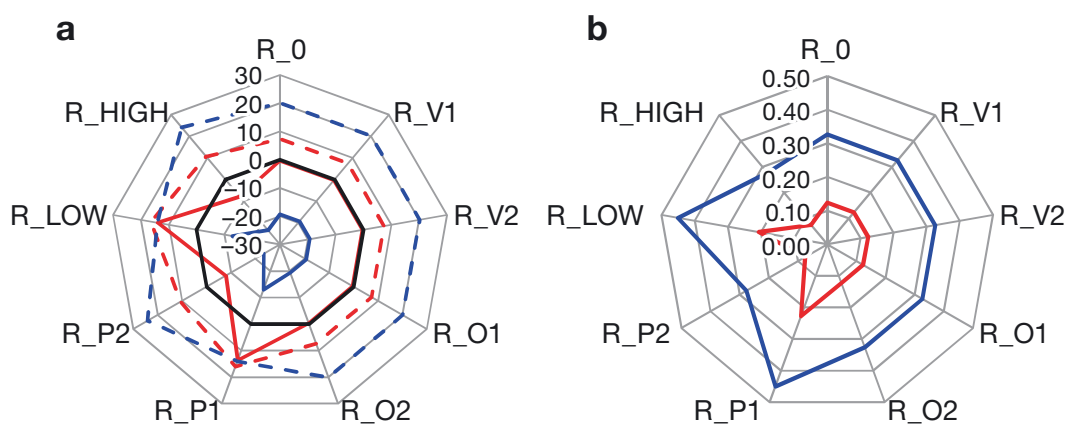

Fig. 3. (a) Two-dimensional (radar) chart of the mean bias error (ME; solid lines) and root mean square errors (RSME; dashed lines) for the 9 different sets of vortex-tracker parameters listed in Table 1, with respect to the observed tropical cyclone (TC) counts during the 1995-2010 period. Blue lines show results for the Climate Forecasting System Reanalysis (CFSR) database at the horizontal resolution of $0.5 \times 0.5^{\circ}$ (CFSR0.5); red lines show results using the RegCM model at the horizontal resolution of $36 \mathrm{~km}$ with the $2.5 \times 2.5^{\circ} \mathrm{CFSR}$ data (RegCM_CFSR2.5). (b) As (a), but showing correlations with respect to the observed TC counts 
complete description of each set of criteria is given in Table 1. Note that the dataset for testing the vortex tracking algorithm is from the CFSR database at the horizontal resolution of $0.5 \times 0.5^{\circ}$ (hereinafter CFSR0.5), whereas the downscaling simulations were conducted using the RegCM model at the horizontal resolution of $36 \mathrm{~km}$ with the $2.5 \times 2.5^{\circ} \mathrm{CFSR}$ data (hereinafter RegCM_CFSR2.5). In the downscaling experiments, the CFRS2.5 data was used as input lateral boundary conditions (updated every $6 \mathrm{~h}$ ).

As seen in Fig. 3, both CFSR0.5 and RegCM CFSR2.5 show that the most sensitive parameter in tracking storm centers is the minimum sea level pressure deficit DP. While changing the vorticity and OCS threshold does not change the errors in TC count noticeably, a small change in the DP threshold leads to significant variation in both mean bias and the absolute errors. Of the 9 criteria tested, the R_0 criteria with $\mathrm{DP}=-5 \mathrm{hPa}$, vorticity $\zeta=5 \times 10^{-5} \mathrm{~s}^{-1}$, and OCS $=5 \mathrm{~m} \mathrm{~s}^{-1}$ give the smallest TC count errors and bias for the RegCM_CFSR2.5 dataset (Fig. 3a). The correlation for R_0 is however smaller than that obtained directly detection from the CFSR0.5 dataset (Fig. 3b), indicating that the annual variation of the TC counts detected in RegCM_CFSR2.5 downscaling is less consistent compared to the observed TC counts (cf. also Fig. 4). Although the correlation is highest for the R_P1 criteria $(0.228$, compared to 0.123 for the R_0 criteria) (Fig. 3b), we chose the R_0 criteria for application of our vortex tracking algorithm in RegCM downscaling, because of its smaller mean bias and root mean square errors. Note that vortex tracking in the CFSR0.5 dataset is more sensitive to changes in DP because of its coarser resolution $(\sim 55 \mathrm{~km}$, compared to the $36 \mathrm{~km}$ resolution in the RegCM downscaling). As such, thresholds for

Table 1. Nine different sets of criteria for the vortex tracking algorithm. $\zeta$ : vorticity; OCS: outer circulation wind speed; DP: minimum sea level pressure deficit threshold. Note that the criteria R_LOW and R_HIGH adopt the lowest and highest bounds, respectively, for each of the 3 parameters

\begin{tabular}{|lccc|}
\hline Criterion & $\zeta\left(\mathrm{s}^{-1}\right)$ & OCS $\left(\mathrm{m} \mathrm{s}^{-1}\right)$ & DP $(\mathrm{hPa})$ \\
\hline R_0 & $5 \times 10^{-5}$ & 5 & -5 \\
R_V1 & $1 \times 10^{-5}$ & 5 & -5 \\
R_V2 & $1 \times 10^{-4}$ & 5 & -5 \\
R_O1 & $5 \times 10^{-5}$ & 3 & -5 \\
R_O2 & $5 \times 10^{-5}$ & 7 & -5 \\
R_P1 & $5 \times 10^{-5}$ & 5 & -3 \\
R_P2 & $5 \times 10^{-5}$ & 5 & -7 \\
R_LOW & $1 \times 10^{-5}$ & 3 & -3 \\
R_HIGH & $1 \times 10^{-4}$ & 7 & -7 \\
\hline
\end{tabular}

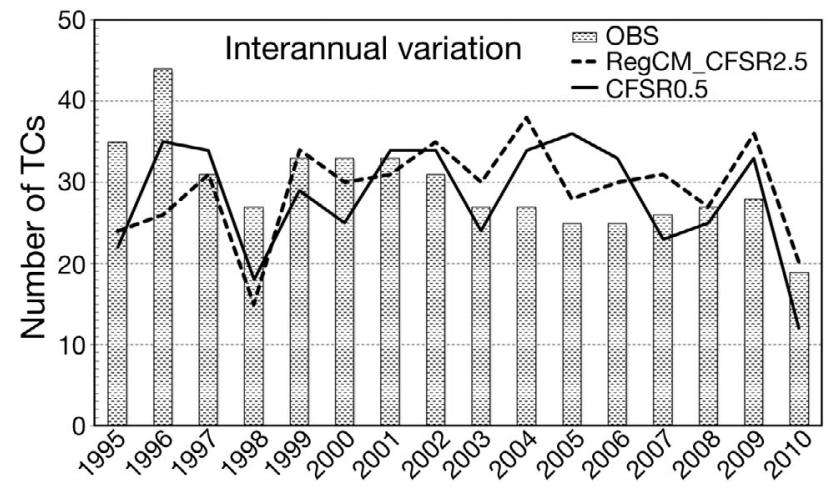

Fig. 4. Interannual TC variation detected from the RegCM_ CFSR2.5 data (dashed lines) and directly from CFSR0.5 (solid lines), using the vortex tracking thresholds R_0 and R_DP0 (see Table 3). Bars: observed TC counts (OBS) during the same period. Further abbreviations as in Fig. 3 legend

CFSR0.5 can be expected to differ from those obtained for the RegCM output. Our sensitivity experiment with further stratification of the pressure deficit threshold DP for tracking vortex centers in the CFSR0.5 dataset shows that the criteria R_DP0 with $\mathrm{DP}=0 \mathrm{hPa}, \zeta=5 \times 10^{-5} \mathrm{~s}^{-1}$, and OCS $=5 \mathrm{~m} \mathrm{~s}^{-1}$ work best for the CFSR0.5 dataset (Table 2). Therefore R_DP0 was selected for all subsequent detection of TCs in the CFSR0.5 data.

Because the criteria R_0 and R_DP0 result in the smallest errors in detecting TC counts for the RegCM downscaling output and CFSR0.5 dataset, these criteria are used next to obtain the total annual TC counts for the entire period 1995-2010 and the spatial distribution of the TC frequency over the entire WPAC basin as given in Figs. 4 \& 5. Notice in Fig. 4 that both the RegCM downscaling and the CFSR0.5 capture the annual variation of TCs well over the entire period, including the most active ENSO phases during 1995-1998, when ENSO transitioned from the La Niña phase (late months of 1995 to late

Table 2. Sensitivity of the mean bias errors (ME), correlation (R) and root mean square errors (RSME) of the vortex tracking algorithm for the CFSR0.5 dataset, with criteria (R_DP0 to R_DP3) defined by different minimum sea level pressure thresholds (DP, hPa), and vorticity and OCS parameters set as for the R_0 criteria in Table 1

\begin{tabular}{|ccccc|}
\hline & DP $(\mathrm{hPa})$ & $\mathrm{ME}$ & $\mathrm{R}$ & $\mathrm{RMSE}$ \\
\hline R_DP0 & 0 & -1.25 & 0.41 & 6.89 \\
R_DP1 & -1 & -2.31 & 0.37 & 7.12 \\
R_DP2 & -2 & -6.88 & 0.47 & 9.21 \\
R_DP3 & -3 & -12.94 & 0.45 & 14.10 \\
\hline
\end{tabular}


1996) to the strong El Niño phase in 1997. Although the correlation of the TC counts from RegCM_ CFSR2.5 is not always high, the difference between the RegCM-detected TC counts and the observed TC counts is within the $95 \%$ confidence interval. Except for the 2001-2004 period, the overall consistent variation of the TC counts obtained from the RegCM downscaling indicates that the new vortex tracking

\section{a Observation}

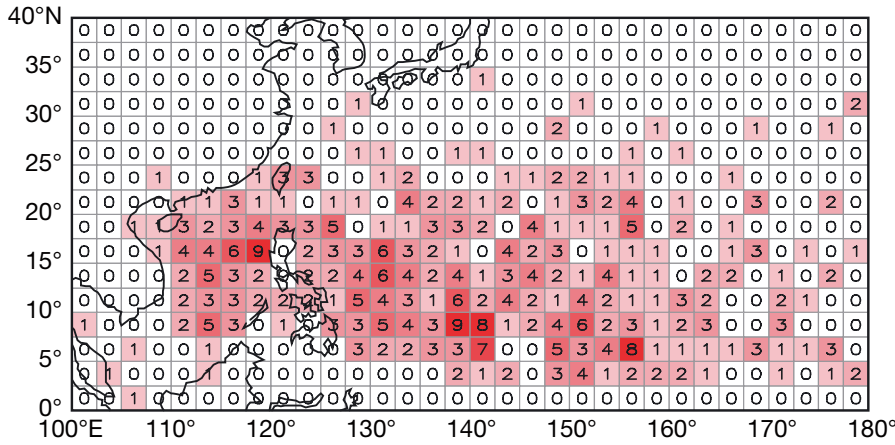

\section{b CFSR}
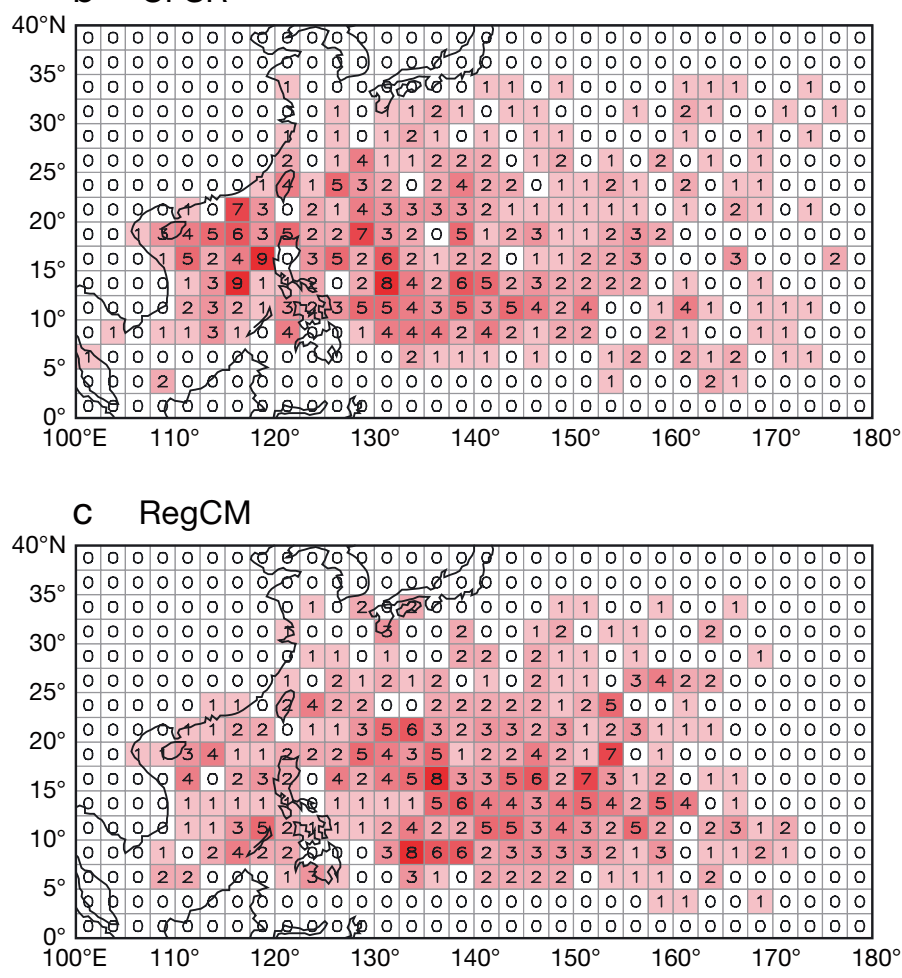

Fig. 5. Spatial distribution of the TC frequency obtained from (a) observation, (b) directly from the CFSR0.5 dataset with the R_DP0 tracking algorithm, and (c) from RegCM_CFSR2.5 outputs using the R_0 tracking algorithm. Numbers in boxes: no. of counts falling within each box; more intense shading: higher number of counts. See Table 3 tracking algorithms and Fig. 3 legend for abbreviations algorithm operates well at the resolution of $36 \mathrm{~km}$, and sufficiently well for subsequent application in the real-time experiment to be presented in Section 4. The TC frequency distribution in Fig. 5 further demonstrates that RegCM_CFSR2.5 captures the overall distribution of the TC activity in the WPAC basin reasonably well. In particular, the elongated region of high TC frequency in the far-east Philippine Sea is well captured in RegCM_CFSR2.5 (i.e. values are similar to observed frequencies), whereas CFSR0.5 captures more TC activity near Philippines Sea and VES.

To assess the realism of the seasonal TC distribution obtained with our TC tracking algorithm, Fig. 6 compares the seasonal TC distributions for CFSR0.5, RegCM_CFSR2.5 and observation, averaged over the 1995-2010 period. It is seen in Fig. 6 that our tracking algorithm captures well both the number of TCs and their seasonal variation, with the maximum value of $\sim 6$ storms during the most active months from mid-August to September as compared to the average of 7.5 storms observed during this period. In particular, RegCM_CFSR2.5 closely reproduces the variation of TC counts from June to July. The bias of the number of TCs between model and observation is acceptable, and it is persistent across the months. A similar tracking algorithm applied directly for the CFSR0.5 dataset underestimates the total storm count in almost months of the year, except in the early season from April to June. Although adjustment of the OCS or the DP criterion could produce better distribution of the monthly TC counts, the impacts of the coarse resolution are still fairly signifi-

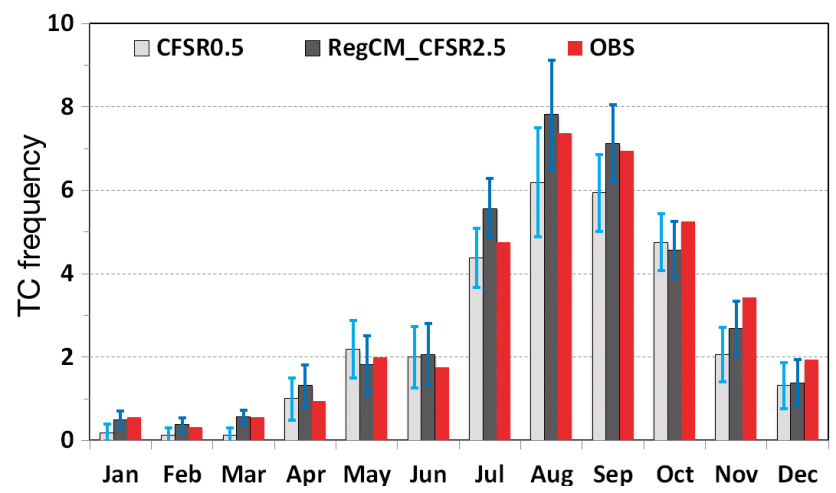

Fig. 6. Seasonal variation of the averaged TC frequency during the 1995-2010 period in the North Western Pacific basin for observed TC count (red), the RegCM_CFSR2.5 simulations (dark gray), and the CFSR0.5 analysis (light gray). Error bars: $95 \%$ confidence intervals for each individual month, which are derived from the 1995-2010 statistics. Further abbreviations as in Fig. 3 legend 
cant, with overall fewer TC counts during this period regardless of OCS or vorticity criteria used in the tracking algorithm, thus indicating the critical role of the grid resolution in capturing TC activity.

Comparison of the geographical distribution of the TC tracks detected in RegCM_CFSR2.5 dataset to the observed tracks during the 1995-2010 period (Fig. 7) reveals further that the RegCM downscaling captures the pattern and lifetime of TCs during this baseline period reasonably well. Regardless of the tracking thresholds, most of the RegCM storms are located in the northern latitudes (north of $5^{\circ} \mathrm{N}$ ) and west of $150^{\circ} \mathrm{E}$ with the overall movement in the southeast to northwest direction at lower latitudes and TCs gradually heading north as they approach the continent. However, the RegCM storms are relatively short-lived compared to the observed TCs during the same period. This is likely because the CFSR2.5 dataset does not contain sufficient cyclonic motion at the $2.5^{\circ}$ horizontal resolution for the RegCM model to enhance further, even after adjusting the vortex searching criteria. Note that there are instances where the model storm centers are still detected over land; these remnants of the model storms after making landfall. While such vortex centers over land could be eliminated entirely by imposing some further check based on the surface landmask, this would affect some cases in which a model storm does maintain its strength over land, even after making landfall, and it is difficult to remove entirely. Because our main focus of the TC seasonal forecasting is on the number of TCs formed over ocean rather than following their entire lifecycle, any subsequent extension of the track over land will not generally impact the count of TCs. However, to ensure that a newly detected storm must form over ocean, any vortex center that is detected over land at the first instance is eliminated, because this indicates that the storm is not a real tropical cyclone.

Given the reasonable performance of the above tracking criteria in our experiment with the RegCM downscaling during the 1995-2010 period, we hereinafter use the R_0 criteria listed Table 1 to detect TC centers in our experimental forecasts of TC frequency in the WPAC basin, using the RegCM model to downscale the $1 \times 1^{\circ}$ CFS real-time forecasts (hereinafter RegCM_CFS1.0).

\section{SEASONAL TC FORECAST FOR WPAC AND VIETNAM AREAS}

\subsection{Real-time TC frequency for WPAC}

To evaluate first the performance of the RegCM_CFS1.0 model in seasonal TC forecasts for the WPAC basin during 2012-2013 seasons, Fig. 8 compares the statistics of the monthly TC counts detected from RegCM outputs to the observed TC counts from February to May in 2012 and 2013. Because the TC season in VNC typically ranges from June to November every year, the analysis in this study will consider only the 6 mo forecasts issued during the February to May period for consistency with the analysis for VNC in the next section.

Since our seasonal forecasts are cycled every $7 \mathrm{~d}$, four 6 mo forecasts

\begin{abstract}
are initiated in each month. The per-
\end{abstract}
Fig. 7. (a) Simulated storm tracks detected from the RegCM_CFSR2.5 experiments (see Fig. 3 legend) with the new vortex tracking algorithm, and (b) observed tracks during the 1995-2010 period. Red star: Hanoi 


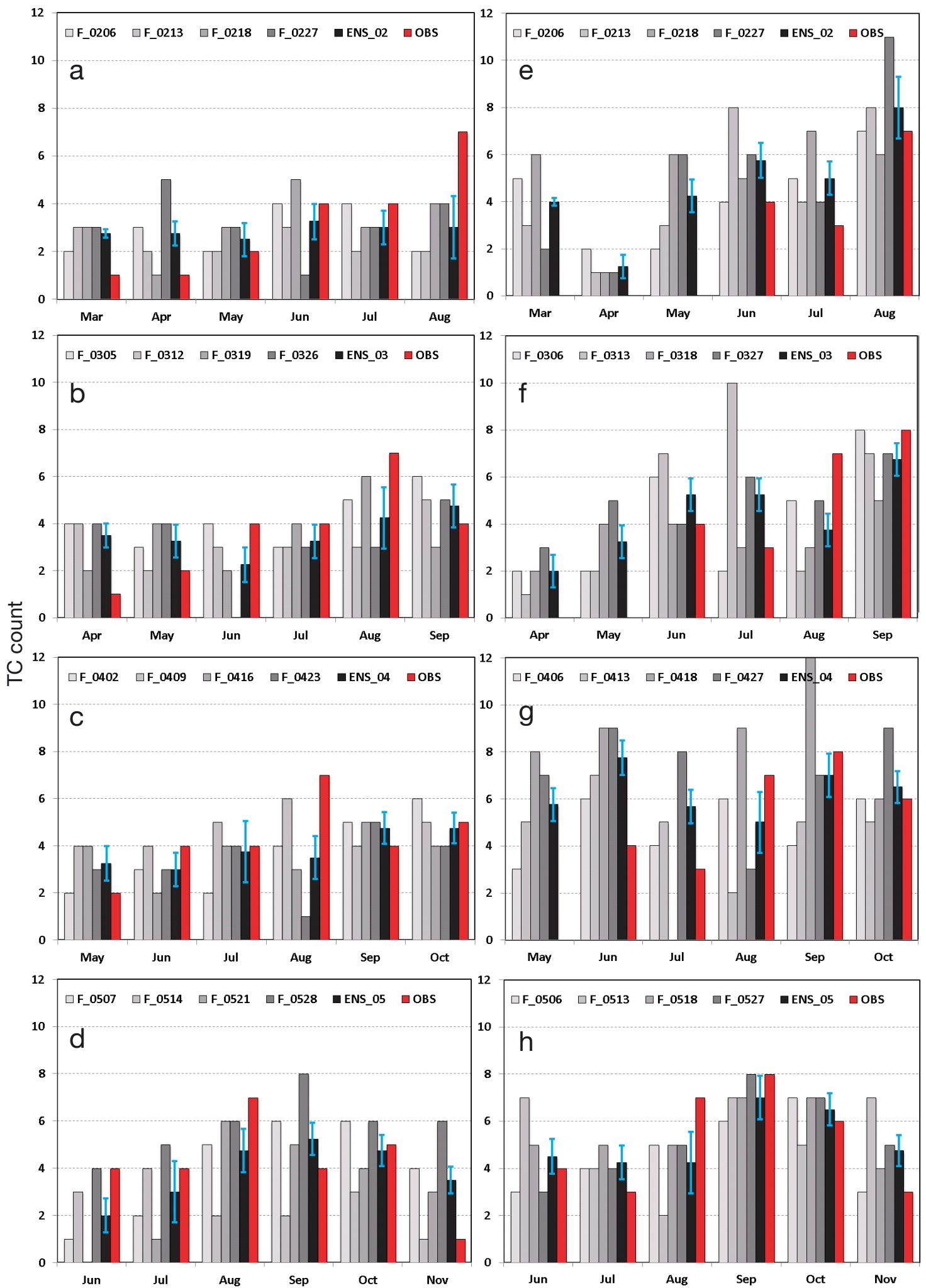

Fig. 8. (a-d) Four 6 mo forecast cycles (gray columns) and the monthly mean (black column) of the TC count forecasts from RegCM_CFS1.0 in (a) February, (b) March, (c) April, and (d) May, and the observed TC frequency (red column) during the same period in a 2012 real-time experiment. $(\mathrm{e}-\mathrm{h}) \mathrm{As}(\mathrm{a}-\mathrm{d})$ but for the 2013 season. Error bars: $95 \%$ confidence interval. Abbreviations as in Fig. 3 legend 
formance of RegCM's seasonal forecast is quantified in terms of monthly TC counts, calculated as the average of the 4 weekly TC count forecasts initiated in a given month. For example, a forecast of the TC count for March that is issued in February is the mean of the $4 \mathrm{TC}$ count forecasts for March from cycles initiated in February.

As seen in Fig. 8, there is significant variation in the storm counts from month to month in RegCM's seasonal forecasts and between the 2012 and 2013 seasons. The variation is relatively small for the weekly cycles initiated in February and March 2012 and then gradually increases in the later months toward the summer with maximum variability occurring in May. For example, the 4 cycles started in May 2012 display different numbers of TC counts of up to 6 storms between the cycle initiated at 00:00 UTC $h$ 14 May and that initiated at 00:00 h UTC 28 May 2012. In contrast, forecasts in 2013 exhibit some specific issues with a significant overestimation of the TC activity in February and March forecast compared with the $95 \%$ confidence interval. The larger variation of TC counts towards summer time appears to be consistent with less predictable conditions in the large-scale region environment, as a result of increasingly energetic summer monsoon activities (Webster et al. 1998, Taraphdar et al. 2010). This is particularly apparent in WPAC, where $>80 \%$ of TCs are related to the Inter Tropical Convergence Zone (ITCZ) (Gray 1968). As such, any variation in the strength or the pattern of the ITCZ could greatly impact the seasonal predictability of TC activity in this area.

Despite larger variation from cycle to cycle, it is of interest that RegCM is able to forecast the general distribution of the TC counts fairly well, showing an upward trend of more TCs towards the summer months as in the observation data. Fig. 9 shows RegCM_CFS1.0's 6 mo forecasts of the total number of TCs issued every month from February to May compared to the observation data for the forecast period (i.e. February forecasts are compared with observation data for March to August). As seen in this bulk statistics, RegCM_CFS1.0 predicts an increasing tendency of TC activity from February to April for both seasons of 2012 and 2013. Although the predicted peaks of the TC counts, in both 2012 and 2013, are somewhat larger than observed (cf. Fig. 8), the consistent trend of the total TC counts within the 6 mo window indicates that the model is capable of developing some basic features of TC distribution.

While the good performance of RegCM_CFS1.0 in seasonal forecasts of TC counts could be attributed to

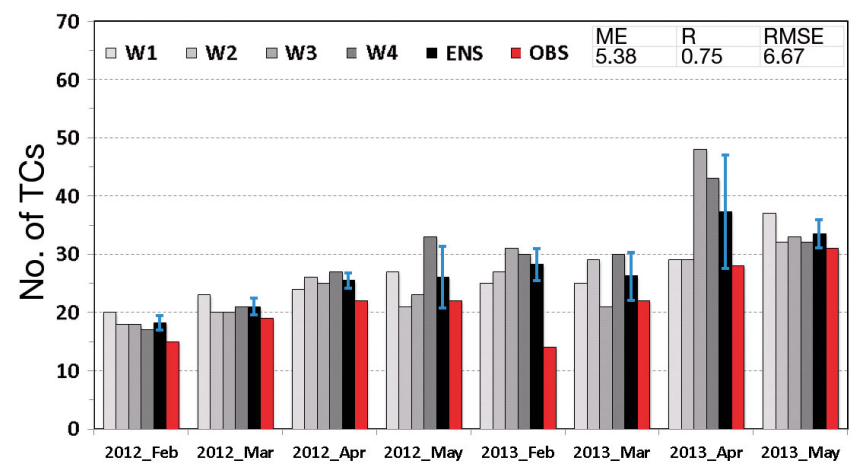

Fig. 9. Comparison the total number of TCs obtained from 4 real-time 6 mo forecast cycles (gray columns) and the monthly mean forecast (black) from the RegCM_CFS1.0 issued from February to May in 2012 and 2013 with the total number of TCs observed (red) in the respective 6 mo forecast periods. W1-4: forecast cycles initialized at the first, second, third, and fourth week of each month, respectively. Error bars: $95 \%$ confidence interval derived from the monthly averaged forecasts. Abbreviations as in Fig. 3 legend and Table 2

its higher resolution, this result could also be influenced by lateral boundary conditions provided by the CFS products. Thus, a good seasonal forecast cannot be entirely attributed to the RegCM model but is to some degree a result of good CFS forecasts. In order to examine the capability of the CFS model in real-time forecasts of TC frequency relative to the RegCM model, Fig. 10 shows the total number of TCs forecasted within the 6 mo interval obtained directly from the CFS1.0 during the 2012 and 2013 seasons. Similar to the forecasts of TC counts in RegCM CFS1.0, the TC count obtained from the CFS1.0 forecasts for any month is an average of the four 6 mo forecasts issued in that month.

Of interest in CFS1.0's 6 mo forecasts of the total number of TCs (Fig. 10) is that CFS1.0 substantially underestimates TC activity throughout the 20122013 seasons, with a maximum TC count of only 3 storms over the entire WPAC basin for forecasts issued in February 2012 as compared to 15 storms observed from March to August 2012. Forecasts in March 2012 do not even capture a single TC during the entire 6 mo lead time. Similar results are seen for 2013 (Fig. 10a). In contrast, RegCM_CFS1.0 shows more realistic number of TCs with the total number of TCs in any month very close to the observed numbers (cf. Figs. 8 \& 9). This comparison is of course not really 'fair' because detecting TC centers directly from the CFS forecasts at a resolution of $1 \times 1^{\circ}$ degree may be sensitive to criteria in the tracking algorithm as discussed in Section 3 (cf. Fig. 3). To address this issue, an additional sensitivity experiment is con- 


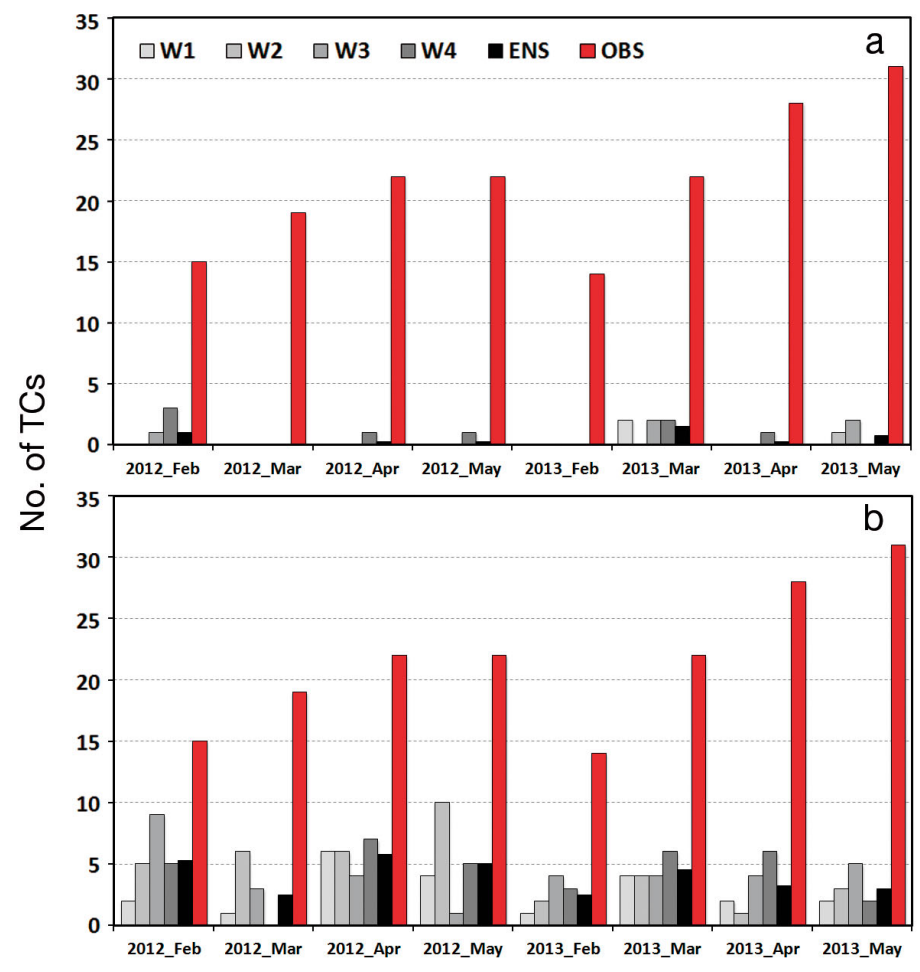

Fig. 10. (a) As Fig. 9, but showing results for the Climate Forecasting System forecasts. (b) As (a), but with a lower vorticity criterion in the vortex tracking algorithm (see text for details)

ducted in which both the vorticity maximum and the OCS value are re-tuned to search for the best number of TCs from the CFS forecasts. The aim of this tuning is to match the criteria to the lower resolution of the CFS products compared to the RegCM model outputs. As seen in Fig. 10b, retuning the searching vortex criteria could help detect 80 to $90 \%$ more TCs in the CFS forecasts during both the 2012 and 2013 seasons. However, the total TC counts are in general still much lower compared to observation or the RegCM_CFS1.0 forecasts. In this regard, the better performance of the RegCM model in seasonal TC forecasts suggests that higher-resolution regional models are still important in enhancing the TC representation and development, which the coarse resolution global forecasts could not attain. In addition to enhancing the capability of the CFS forecast, regional downscaling is useful as it allows examination of different climate change scenarios driven by the global changes, not only in terms of TC count but also changes in the track patterns and genesis frequency that are not fully captured by global models.

Two experiments were designed to further examine the skill of the RegCM model in the tercile sea- sonal forecasting of the TC frequency with respect to the observed climatology (EXP_1) and model climatology (EXP_2). For these experiments, the observed TC and model TC climatology are obtained from the number of TCs observed during the 1981-2010 period and from the model simulations (RegCM CFSR2.5) during the 1995-2010 period, based on values for the 33rd (observed: q33o; model: q33m) and 66th (q66o; q66m) percentiles (see Phan et al. 2014). For specific evaluation of the tercile forecasts of the TC activity, the number of TCs obtained from RegCM_CFS1.0 (NTCs) during the 2012-2013 seasons is compared against the observed climatology (i.e. q33o and q66o; EXP_1), and against the model climatology (i.e. q33m and q66m; EXP_2) to classify forecasts in below normal (B), normal $(\mathrm{N})$, or above normal (A) categories, where B is defined as NTCs $<\mathrm{q} 33 \mathrm{o}(\mathrm{q} 33 \mathrm{~m}), \mathrm{N}-\mathrm{NTCs}$ are in between q33o (q33m) and q66o (q66m), and A-NTCs > q66o (q66m) (Table 3); the corresponding statistical scores are provided in the Table 4.

As seen in Fig. 11, while the 2012 season shows normal activity, with all 6 mo forecasts falling within the q33m-q66m range in EXP_2 and 3 of 4 falling within the q33o-q66o range in EXP_1, the 2013 season exhibits predominantly above-normal activity, especially toward March to May months, which explains the fact that above-normal forecasts (A) have the overall highest bias scores in Table 4. As a result, the absolute error in the TC count forecasts in 2013 is substantially higher than in 2012, as seen in Fig. 11. Values of the bias and probability of detection score for category $\mathrm{B}$ and $\mathrm{N}$ forecasts are rather

Table 3. RegCM real-time tercile forecasts of the total number of TCs during the 2012-2013 seasons for categories of below normal (B), normal (N), and above normal (A). These categories are defined with respect to the 33 and $66 \%$ percentiles obtained from the observed climatology (EXP_1) and model climatology (EXP_2) of TC activity during the baseline periods 1981-2010 and 1995-2010, respectively

\begin{tabular}{|lccccc|}
\hline Experiment & OBS & B & N & A & SUM \\
& Forecast & & & & \\
\hline EXP_1 & B & 1 & 0 & 0 & 1 \\
& N & 1 & 5 & 1 & 7 \\
& A & 2 & 11 & 11 & 24 \\
& SUM & 4 & 16 & 12 & 32 \\
EXP_2 & B & 1 & 0 & 0 & 1 \\
& N & 1 & 5 & 0 & 6 \\
& A & 2 & 11 & 12 & 25 \\
& SUM & 4 & 16 & 12 & 32 \\
\hline
\end{tabular}



May in 2012 and 2013. To be specific, any TC whose

Fig. 12. As Fig. 9 but for Vietnam's coastal region within a domain of $\left(100-120^{\circ} \mathrm{E}\right) \times\left(5-25^{\circ} \mathrm{N}\right)$
Table 4. Verification scores of model forecasts of TC activity with respect to observed climatology (EXP_1) and model climatology (EXP_2) based on data in Table 3, showing bias scores, with bias categorized as below normal (B), normal (N), and above normal (A), and probability of detection (POD) for each category. Values for proportion correct (PC), Heidke Skill Score (HSS), and Peirce Skill Score (PSS) are for all model forecasts

\begin{tabular}{|llllllllll|}
\hline $\begin{array}{l}\text { Score } \\
\text { EXP Bias_B }\end{array}$ & \multicolumn{1}{l}{ Bias_N Bias_A POD_B } & POD_N & POD_A & PC & HSS & PSS \\
\hline EXP_1 & 0.25 & 0.44 & 2.00 & 0.25 & 0.31 & 0.92 & 0.53 & 0.226 & 0.230 \\
EXP_2 & 0.25 & 0.38 & 2.08 & 0.25 & 0.31 & 1.00 & 0.56 & 0.282 & 0.289 \\
\hline
\end{tabular}
the equitable forecasts during both seasons, with HSS values of 0.226 and 0.282 , and PSS values of 0.230 and 0.289 for the EXP_1 and EXP_2, respectively (see Tables $3 \& 4$ ). Such positive scores are attributed mostly to the ability of the RegCM model in detecting correctly the above-normal TC counts in all forecast cycles from March-June 2013. Furthermore, the fact that values of the HSS and PSS in EXP_2 are somewhat larger in EXP_1 suggests that for the tercile forecast, the model climatology should be used instead of the observed one.

It is encouraging to see that RegCM also captures well both the tendency of above-normal activity in 2013 and the normal activity in 2012. If the phase of the anomaly forecast is used to quantify the performance of the tercile forecast, it is seen from Fig. 11 (and also from Table 3) that RegCM has 17 correct phase forecasts out of 32 in EXP_1 and 18 out of 32 in EXP_2, corresponding to proportion correct (PC) scores of 0.53 and 0.56 respectively. In contrast, CFS provides well below normal activity in all forecasts, with all of the tercile forecasts below the normal climatology (Fig. 11). Such consistent phase forecast in RegCM again suggests that RegCM is capable of correctly reproducing the TC anomaly tendency that the global CFS forecasts cannot achieve.

\subsection{Seasonal forecasts for Vietnam's coastal region}

To focus further on the seasonal TC forecasts for the VNC area, this subsection examines forecasts of TCs whose gale force winds and associated circulation potential threaten Vietnam's coastline. Fig. 12 plots the number of TCs detected in the VNC region (rather than for the entire WPAC basin) during periods covered by forecasts issued from February to

small while they are quite
large for category A forecasts (Table 4), reflecting missed phases, and false alarms in the 'A' phase of the model. Despite the overestimation of the TC count in 2013, direct calculation of the Heidke Skill Score (HSS) or Peirce Skill Score (PSS) for these 3-category phase forecasts appears to confirm some from RegCM (green symbols for the weekly forecasts [W1-4], blue circles for the ensemble means), and CFS forecasts and the observation ata (red circles) for the correspont 6 mo forecast periods. Red lines show the $33 \%$ (dashed) and ogy of TC activity during the 1981-2010 baseline period. Blue lines show the corresponding 33\% (dashed) and 66\% (solid) percentiles obtained from the model climatology con2010 baseline period

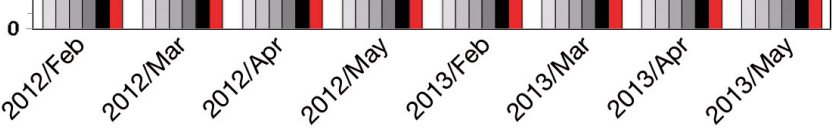


center is within a domain of $\left(5\right.$ to $\left.25^{\circ} \mathrm{N}\right) \times(100$ to $120^{\circ} \mathrm{E}$ ) during any stage of its lifetime is considered to have potential influence on Vietnam's coastline. This definition thus includes storms that may form in the far ocean but later enter the selected domain. The number of TCs in this sub-region is overall too small to give a statistically significant result, but sufficient to see if the trend of the TC distribution is reflected in RegCM_CFS1.0's forecasts for this area.

As seen in Fig. 12, RegCM_CFS1.0, the 6 mo forecasts issued from February to May greatly overestimate the total number of TCs in the VNC area for both the 2012 and 2013 seasons compared with the $95 \%$ confidence intervals. Unlike the forecast for the whole WPAC region, the TC activity in VNC fluctuates markedly from cycle to cycle due to the small number of the TCs in this area, with an average of 10 to 12 TCs for most of the 6 mo forecasts. In contrast, observation consistently shows a smaller number of TCs ( $\sim 7$ to 9$)$. Although the number of the observed TCs over the entire WPAC basin is larger than that obtained from RegCM_CFS1.0's forecasts (Fig. 9), many observed TCs did not enter the VES but instead shifted in a north-northwesterly direction, similarly to in the baseline period (cf. Fig. 7). As a result, the observed number of TCs that actually entered the VES and subsequently impacted Vietnam's coastline is considerably smaller than the total observed count during the entire period. That the TC counts obtained from the RegCM_CFS1.0 are similar to the observed counts in the WPAC basin but much greater than observed counts in the VNC area suggests that RegCM_CFS1.0 has some potential issues with the large-scale flows that, in the model, somehow expand too far to the west and veer towards the VES instead of turning to the north as observed.

\section{CONCLUSIONS}

We have presented experimental seasonal forecasts of tropical cyclone (TC) activity for Vietnam's coastal region during the 2012-2013 typhoon seasons, using the RegCM4.2 to downscale the global CFS forecasts (RegCM_CFS1.0). By optimizing Walsh's (1997) vortex tracking algorithm, by giving more weight to the magnitude of the outer core wind and imposing some empirical thresholds on the vorticity criterion, we developed a modified tracking algorithm capable of detecting model vortex centers very well in the CFSR dataset for a baseline period from 1995-2010. Model simulations obtained are consistent with observation with reference to the total TC counts, the monthly variations in TC frequency, and the TC track pattern.

Application of the new tracking algorithm to the real-time 6 mo forecasts of TC frequency during the 2012-2013 seasons with the RegCM_CFS1.0 showed that RegCM can predict TCs fairly well in the Northwestern Pacific (WPAC) basin in terms of both the magnitude and the distribution of TC frequency as compared to the observed TC distribution. Except for the February and March forecasts in 2013, RegCM_CFS1.0 consistently captured the total number of TCs during the experimental period with $\sim 15$ to $20 \%$ more TCs towards summer time (April to May) compared to forecasts issued in the February-March period. Although part of RegCM's good performance in forecasting the TC frequency is inherited from the good quality of the CFS forecast, examination of the total TC counts directly obtained from the CFS forecasts revealed that the CFS forecasts do not capture TC frequency during the 2012-2013 seasons. Experiments with different tracking thresholds showed that underestimation of the TC count in the CFS forecast is an inherent feature of this global product, and is a consequence of the low resolution and possibly simplified physics of the CFS model. Thus, the ability of the RegCM model in both enhancing TC representation and reflecting observed distribution of the TC counts demonstrates the importance of the regional models in seasonal forecasting of TC activity. Further analysis of the total TC counts for the VNC area showed, however, that RegCM tends to overestimate the TC frequency in this sub-region, despite giving good forecasts for the whole WPAC basin. While the results obtained in this real-time experiment are not conclusive due to limited sample size, our plan is to continue the real-time experiments in order to generate more robust statistics. The possibility of increasing the horizontal resolution of the regional climate model system to allow for more detailed analysis will be explored and presented in an upcoming study.

Acknowledgments. This research was supported by the Vietnam Ministry of Science and Technology Foundation under the Project No: DT.NCCB-DHUD.2011-G/09. This work was also encouraged and partially supported by the 11-P04-VIE Danida Project. We express our sincere thanks to 3 anonymous reviewers, the Editor, and Prof. Roger K. Smith, whose comments and suggestion have helped to substantially improve this manuscript. 


\section{LITERATURE CITED}

Becker EJ, Van Den Dool H, Peña M (2013) Short-term climate extremes: prediction skill and predictability. J Clim 26:512-531

Bengtsson L, Botzet M, Esch M (1995) Hurricane type vortices in a general circulation model. Tellus Ser A Dyn Meterol Oceanogr 47:175-196

Bengtsson L, Hodges KI, Esch M (2007) Tropical cyclones in a T159 resolution global climate model: comparison with observations and re-analyses. Tellus 59A:396-416

Camargo SJ, Barnston AG (2009) Experimental seasonal dynamical forecasts of tropical cyclone activity at IRI. Weather Forecasting 24:472-491

Camargo SJ, Zebiak SE (2002) Improving the detection and tracking of tropical storms in atmospheric general circulation models. Weather Forecast 17:1152-1162

Chan JCL (2008) A simple seasonal forecast update of tropical cyclone activity. Weather Forecast 23:1016-1021

Chan JCL, Shi JE, Lam CM (1998) Seasonal forecasting of tropical cyclone activity over the western North Pacific and the South China Sea. Weather Forecast 13:997-1004

Chan JCL, Shi JE, Liu KS (2001) Improvements in the seasonal forecasting of tropical cyclone activity over the western North Pacific. Weather Forecast 16:491-498

Dickinson RE, Errico RM, Giorgi F, Bates GT (1989) A regional climate model for the western United States. Clim Change 15:383-422

Elguindi N, Bi X, Giorgi F, Nagarajan B, Pal J, Solmon F, Zakey A (2004) RegCM version 3.0 user's guide. The Abdus Salam International Centre for Theoretical Physics (ICTP), Trieste

Elguindi N, Bi X, Giorgi F, Nagarajan B, Pal J, Solmon F, Giuliani G (2011) Regional climatic model RegCM user manual version 4.1. ICTP, Trieste

Gillies RR, Wang SY, Yoon JH, Weaver S (2010) CFS prediction of winter persistent inversions in the intermountain region. Weather Forecast 25:1211-1218

Giorgi F, Anyah RO (2012) The road towards RegCM4. Clim Res 52:3-6

> Giorgi F, Bates GT (1989) The climatological skill of a regional model over complex terrain. Mon Weather Rev 117:2325-2347

Giorgi F, Marinucci MR, Bates GT (1993a) Development of a second-generation regional climate model (RegCM2). I. Boundary-layer and radiative transfer processes. Mon Weather Rev 121:2794-2813

Giorgi F, Marinucci MR, Bates GT, De Canio G (1993b) Development of a second-generation regional climate model (RegCM2). II. Convective processes and assimilation of lateral boundary conditions. Mon Weather Rev 121:2814-2832

Giorgi F, Coppola E, Solmon F, Mariotti L and others (2012) RegCM4: model description and preliminary tests over multiple CORDEX domains. Clim Res 52:7-29

Gray WM (1968) Global view of the origin of tropical disturbances and storms. Mon Weather Rev 96:669-700

Grell GA, Dudhia J, Stauffer D R (1994) A description of the fifth-generation Penn State/NCAR mesoscale model (MM5). Technical Note NCAR/TN-398+STR, National Center for Atmospheric Research (NCAR), Boulder, $\mathrm{CO}$

Kim JH, Ho CH, Kim HS, Choi W (2012) 2010 Western North Pacific typhoon season: seasonal overview and forecast using a track-pattern-based model. Weather Forecast 27: 730-743

Kirtman BP, Min D (2009) Multimodel ensemble ENSO prediction with CCSM and CFS. Mon Weather Rev 137: 2908-2930

Klotzbach PJ (2007) Revised prediction of seasonal Atlantic basin tropical cyclone activity from 1 August. Weather Forecast 22:937-949

Klotzbach PJ, Gray WM (2009) Twenty-five years of Atlantic basin seasonal hurricane forecasts (1984-2008). Geophys Res Lett 36:L09711, doi:10.1029/2009GL037580

Knapp KR, Kruk MC (2010) Quantifying interagency differences in tropical cyclone best-track wind speed estimates. Mon Weather Rev 138:1459-1473

Lu MM, Chu PS, Lin YC (2010) Seasonal prediction of tropical cyclone activity near Taiwan using the Bayesian multivariate regression method. Weather Forecast 25:1780-1795

> Lu MM, Lee CT, Wang B (2013) Seasonal prediction of accumulated tropical cyclone kinetic energy around Taiwan and the sources of the predictability. Int J Climatol 33: 2846-2854

Nguyen KC, Walsh KJE (2001) Interannual, decadal, and transient greenhouse simulation of tropical cyclone-like vortices in a regional climate model of the South Pacific. J Clim 14:3043-3054

Pal JS, Giorgi F, Bi X, Elguindi N and others (2007) Regional climate modeling for the developing world: the ICTP RegCM3 and RegCNET. Bull Am Meteorol Soc 88: 1395-1409

> Phan VT, Ngo-Duc T, Ho TMH (2009) Seasonal and interannual variations of surface climate elements over Vietnam. Clim Res 40:49-60

Phan VT, Nguyen VH, Trinh TL, Nguyen QT, Ngo-Duc T, Laux P, Nguyen XT (2014) Seasonal prediction of surface air temperature across Vietnam using the Regional Climate Model version 4.2 (RegCM4.2). Adv Meteorol 2014: 245104, doi:10.1155/2014/245104

> Saha S, Moorthi S, Pan HL, Wu X, Wang J, Nadiga S, Reynolds RW (2010) The NCEP climate forecast system reanalysis. Bull Am Meteorol Soc 91:1015-1057

Solmon F, Mallet M, Elguindi N, Giorgi F, Zakey AS, Konare A (2008) Dust aerosol impact on regional precipitation over western Africa, mechanisms and sensitivity to absorption properties. Geophys Res Lett 35:L24705, doi: 10.1029/2008GL035900

Sooraj KP, Annamalai H, Kumar A, Wang H (2012) A comprehensive assessment of CFS seasonal forecasts over the tropics. Weather Forecast 27:3-27

> Strachan J, Vidale PL, Hodges K, Roberts M, Demory ME (2013) Investigating global tropical cyclone activity with a hierarchy of AGCMs: the role of model resolution. J Clim 26:133-152

- Taraphdar S, Mukhopadhyay P, Goswami BN (2010) Predictability of Indian summer monsoon weather during active and break phases using a high resolution regional model. Geophys Res Lett 37:L21812, doi:10.1029/2010 GL044969

Vecchi GA, Delworth T, Gudgel R, Kapnick S and others (2014) On the seasonal forecasting of regional tropical cyclone activity. J Clim 27:7994-8016

Vecchi GA, Zhao M, Wang H, Villarini G and others (2011) Statistical-dynamical predictions of seasonal North Atlantic hurricane activity. Mon Weather Rev 139:1070-1082 Vitart F, Leroy A, Wheeler MC (2010) A comparison of 
dynamical and statistical predictions of weekly tropical cyclone activity in the Southern Hemisphere. Mon Weather Rev 138:3671-3682

Walsh K (1997) Objective detection of tropical cyclones in high-resolution analyses. Mon Weather Rev 125: 1767-1779

Walsh KJ, Ryan BF (2000) Tropical cyclone intensity increase near Australia as a result of climate change. J Clim 13:3029-3036

- Walsh K, Watterson IG (1997) Tropical cyclone-like vortices in a limited area model: comparison with observed climatology. J Clim 10:2240-2259

> Wang W, Chen M, Kumar A (2010) An assessment of the CFS real-time seasonal forecasts. Weather Forecast 25:

Editorial responsibility: Filippo Giorgi,

Trieste, Italy
950-969

Weatherford CL, Gray WM (1988) Typhoon structure as revealed by aircraft reconnaissance. I. Data analysis and climatology. Mon Weather Rev 116:1032-1043

Webster PJ, Magana VO, Palmer TN, Shukla J, Tomas RA, Yanai MU, Yasunari T (1998) Monsoons: processes, predictability, and the prospects for prediction. J Geophys Res 103:14451-14510.

Wu R, Kirtman BP, van den Dool H (2009) An analysis of ENSO prediction skill in the CFS retrospective forecasts. J Clim 22:1801-1818

Yeung AY, Chan JC (2012) Potential use of a regional climate model in seasonal tropical cyclone activity predictions in the western North Pacific. Clim Dyn 39:783-794

Submitted: October 17, 2013; Accepted: October 10, 2014 Proofs received from author(s): December 26, 2014 